\title{
Optimization of Microencapsulation of Inonotus obliquus Extract Powder by Response Surface Methodology and Its Application into Milk
}

\author{
Sung-II Ahn, Yoon-Hyuk Chang, and Hea-Soo Kwak* \\ Department of Food Science and Technology, Sejong University, Seoul 143-747, Korea
}

\begin{abstract}
This study was carried out to optimize microencapsulation conditions for Inonotus obliquus extract powder with mediumchain triacylglycerol (MCT) using response surface methodology (RSM), and to determine the possibility of adding encapsulated I. obliquus extract to milk toward improving the functionality of milk. and to investigate the effects of added encapsulated I. obliquus extract on the physicochemical and sensory properties of the product. The microencapsulation efficiency of I. obliquus extract powder was investigated with respect to three variables (ratios of core material to coating material, amounts of water added, and spray pressure) in RSM. The optimal conditions for microencapsulation of I. obliquus extract powder were obtained from the ratio of core material to coating material $(2.92: 10)$ and the amount of water added $(0.5 \mathrm{~mL})$; the spray pressure had an insignificant effect on the microencapsulation $(\mathrm{p}<0.05)$. Adding encapsulated I. obliquus extract into milk did not significantly affect either color (L, a and b values) or thiobarbituric acid (TBA) values. Sensory test revealed that there were significant improvements in brownish, herb flavor, and bitterness scores for encapsulated I. obliquus extract-added milk as compared with those for uncapsulated I. obliquus extract-added milk. Based on the data obtained from the present study, it is concluded that microcapsules of I. obliquus extract powder could be applicable to milk without remarkably adverse effects on physicochemical and sensory properties
\end{abstract}

Key words: Inonotus obliquus, microencapsulation, response surface methodology, milk

\section{Introduction}

Inonotus obliquus, also called Chaga mushroom, is a black parasitic fungus that belongs to family Hymenochaetaceae and grows on living trunks of the mature birch. It is widely distributed in North America, Europe, and Asia located at latitudes of $45-50^{\circ} \mathrm{N}$. Traditionally, it has been used for the treatment of gastrointestinal cancer, cardiovascular disease and diabetes since the $16^{\text {th }}$ century in Russia, Poland, and most of Baltic countries (Cui et al., 2005; Hu et al., 2009; Ju et al., 2010; Lee et al., 2007). Moreover, it has been reported that this fungus has the health-promoting functions, such as protection of DNA from the damage induced by oxidative stress (Park et al., 2004), anti-inflammatory (Park et al., 2005), antioxidant (Cui et al., 2005), and antitumor activities (Kahlos et al., 1986). Despite the health benefits of I. obliquus mentioned above, the mushroom has high viscosity, undesir-

\footnotetext{
*Corresponding author: H. S. Kwak, Department of Food Science and Technology, Sejong University, Seoul 143-747, Korea. Tel: 82-2-3408-3226, Fax: 82-2-3408-4319, E-mail: kwakhs@sejong.ac.kr
}

able flavor and color. To overcome the problems, the microencapsulation technique can be a good application for I. obliquus into the food system.

Microencapsulation, a technique for encapsulating tiny particles or droplets by coating materials, has been widely applied in the food industry to mask the core off-taste, hide the core color, prevent oxidation and protect functional materials (Gharsallaoui et al., 2007; Shahidi and Han, 1993). Even though milk is a nutritious food all over the world, some consumers require special functional properties in the milk. Therefore, over the years, many different food ingredients, such as L-ascorbic acid (Lee et al., 2004), isoflavone (Jeon et al., 2005), fish oil (Fonollá et al., 2009), chitooligosaccharide (Choi et al., 2006), and Korean mistletoe extract (Kim et al., 2008) have been microencapsulated in milk formulation to improve the functional value.

To accomplish the microencapsulation of I. obliquus, the most important thing to be considered in the present study was the choice of a coating material to form the microcapsules. In the present study, medium-chain triacylglycerol (MCT) was used as a coating material for the microencapsulation of I. obliquus extract powder because 
this has been proven as an effective coating material in our previous studies (Jeon et al., 2005; Kim et al., 2008; Lee et al., 2004).

So-called healthy foods, especially those with nutraceutical properties, are in great demand in our health conscious society. Nutraceutical milk could be a good vehicle in this respect if nutraceutical ingredients, such as I. obliquus, were to be added into milk. However, there is no report in the literature on the production of milk which incorporates the microencapsulated I. obliquus. Therefore, the objectives of the present study were to optimize the microencapsulation condition for I. obliquus extract powder with MCT using response surface methodology (RSM), and evaluate the effects of adding the microencapsulated I. obliquus extracted into milk on the physicochemical and sensory properties during storage.

\section{Materials and Methods}

\section{Materials}

As a core material, I. obliquus extract powder was provided from Hyun Communication (Seoul, Korea). The coating material, MCT and polyethylene sorbitan monostearate (Tween-60) were obtained from Il-Shin Emulsifier Co., Ltd. (Seoul, Korea). Market milk used was manufactured from Seoul Dairy Co-op (Ansan, Korea).

\section{Experimental design for response surface method- ology}

RSM was employed to optimized the effective process of microencapsulation of I. obliquus powdered extract with respect to operating parameters [ratios of core material to coating material $\left(X_{1}\right)$, amounts of water added $\left(X_{2}\right)$ and spray pressure $\left(X_{3}\right)$ ], which were ranged from $0.5: 10$ to $5.5: 10(\mathrm{w} / \mathrm{w}), 0.5$ to $11.5 \mathrm{~mL}$, and 1,100 to $2,300 \mathrm{psi}$, respectively. The actual variable was coded to facilitate multiple regression analysis (Table 1). The least-square regression model was adequately fitted into

Table 1. Coded levels for independent variables used in experimental design for microencapsulation of Inonotus obliquus extract powder

\begin{tabular}{clccc}
\hline \hline \multirow{2}{*}{$\begin{array}{c}\text { Coded } \\
X_{i}\end{array}$} & \multicolumn{1}{c}{ Variable } & \multicolumn{3}{c}{ Coded level } \\
\cline { 3 - 5 } & & -1 & 0 & 1 \\
\hline$X_{1}$ & $\begin{array}{l}\text { Ratio of coating material } \\
\text { to core material }\end{array}$ & $0.5: 10$ & $3.0: 10$ & $5.5: 10$ \\
$X_{2}$ & Water (mL) & 0.5 & 6.0 & 11.5 \\
$X_{3}$ & Spray pressure (psi) & 1,100 & 1,700 & 2,300 \\
\hline
\end{tabular}

the responses taken from the experimental data and to define an optimization process of the microencapsulation efficiency.

Twenty experimental settings consisting of 6 star points (star distance is 0 ), 6 central points and 8 factorial points were generated with 3 factors and 3 levels by the principal of RSM using MINITAB release 15 (Korean version, Minitab Korea, Gunpo, Korea). The regression model equation for the microencapsulation efficiency (\%) of $I$. obliquus extract powder could be predicted as follows:

Microencapsulation efficiency

$$
=b_{0}+\sum_{i=1}^{3} b_{i} X_{i}+\sum_{i=1}^{3} b_{i i} X_{i}^{2}+\sum_{i=1 j}^{2} \sum_{j=i+1}^{3} b_{i j} X_{i} X_{j}
$$

where subscripts $i$ and $j$ took values from 1 to the number of variables $(n)$; the $b_{0}$ was the intercept term; the $b_{i}$ values were linear coefficient; the $b_{i j}$ values were quadratic coefficient; $X_{i}$ and $X_{j}$ were uncoded independent variables.

\section{Preparation of microcapsule}

The microcapsule was prepared by the method from Kim et al. (2008). I. obliquus extract powder was mixed with water, and the suspension was mixed with MCT for $1 \mathrm{~min}$ at 1,200 rpm with a stirrer. An airless paint sprayer (W-300, Wagner Spray Tech. Co., Markdorf, Germany) nebulized the emulsion of the coating material-I. obliquus extract powder into a cylinder containing a $0.05 \%$ Tween60 solution at $5^{\circ} \mathrm{C}$. The diameter of the nozzle orifice used was $0.4 \mathrm{~mm}$. Microcapsules were formed as lipid solidified in the chilled fluid.

To elucidate the microencapsulation efficiency, total polyphenol contents in I. obliquus extract powder and total polyphenol contents in residue after centrifuging the microcapsules were measured as follows. The chilled fluid was centrifuged at $4,500 \times \mathrm{g}$ for $20 \mathrm{~min}$ to separate the supernatant (microcapsule suspension). After centrifuging, the residue was assayed for untrapped polyphenol contents in I. obliquus extract powder. The total polyphenol contents were evaluated by the Folin-Ciocalteu method as described by Wong and Chye (2009). The microencapsulation efficiency (\%) was calculated as follows:

Microencapsulation efficiency $(\%)=[1-($ total polyphenol contents in residue/total polyphenol contents in I. obliquus extract powder) $] \times 100$ 


\section{Microscopic observation}

The microstructural image of the microcapsule was magnified by 1,000-folds with a light microscope (Eclipse 80i, Nikon, Tokyo, Japan). The measurement of capsule sizes was employed by Dixi imaging solution Ver. 2.89 (Dixioptics, Seoul).

\section{Supplementation of microencapsulted or uncapsu- lated I. obliquus extract into milk}

Encapsulated or uncapsulated I. obliquus extract was aseptically added into pasteurized milk (1\%, v/v) on the basis of the preliminary study. Each milk sample was stored for 0,3 , and $6 \mathrm{~d}$ at $7^{\circ} \mathrm{C}$ to evaluate $\mathrm{pH}$, color, TBA, and sensory properties as a short period of storage.

\section{pH analysis}

The $\mathrm{pH}$ values of each milk sample were measured at $7^{\circ} \mathrm{C}$ using a pH meter (Orion 900A, Boston, MA, USA).

\section{Color measurement}

Color values of each milk sample were investigated using a colorimeter (CR210; Minolta, Tokyo, Japan) after calibrating its original value with a standard plate ( $X=97.83, Y=81.58, Z=91.51)$. Measured CIE L*-, a*-, and $b^{*}$-values were used as indicators of lightness, red- ness and yellowness, respectively, in artificial light. This was triplicated.

\section{Thiobarbituric acid (TBA) test}

The absorbance changes by addition of encapsulated or uncapsulated $I$. obliquus extract into milk was measured using a thiobarbituric acid (TBA) test, as described by Stapelfeldt et al. (1997).

\section{Sensory analysis}

Eight-trained sensory panelists evaluated randomly coded milk samples. The color, rancidity, bitterness, astringency, and overall acceptability were investigated on a 7-point scale ( $1=$ very weak, $4=$ moderate, $7=$ very strong).

\section{Statistical analysis}

All statistical analyses were performed using SAS version 9.0 (SAS Institute Inc., Cary, NC, USA). Analysis of variance (ANOVA) was performed using the general linear models (GLM) procedure to determine significant differences among the samples. Means were compared by using Fisher's least significant difference (LSD) procedure. Significance was defined at the $5 \%$ level. This was triplicated.

Table 2. Central composite design for the optimization of microencapsulation of Inonotus obliquus extract powder using medium-chain triacylglycerol as a coating material

\begin{tabular}{|c|c|c|c|c|c|c|c|}
\hline \multirow{2}{*}{ Run number } & \multicolumn{3}{|c|}{ Coded variable $^{1}$} & \multicolumn{3}{|c|}{ Process variable } & \multirow{2}{*}{$\begin{array}{l}\text { Microencapsulation } \\
\text { efficiency }(\%)\end{array}$} \\
\hline & $X_{1}$ & $X_{2}$ & $X_{3}$ & $X_{1}$ & $X_{2}$ & $X_{3}$ & \\
\hline 1 & 1 & 1 & -1 & $5.5: 10$ & 11.5 & 1100 & 92.87 \\
\hline 2 & -1 & -1 & -1 & $0.5: 10$ & 0.5 & 1100 & 93.05 \\
\hline 3 & -1 & 1 & 1 & $0.5: 10$ & 11.5 & 2300 & 86.63 \\
\hline 4 & 1 & -1 & 1 & $5.5: 10$ & 0.5 & 2300 & 92.17 \\
\hline 5 & 0 & 0 & 0 & $3.0: 10$ & 6.0 & 1700 & 93.25 \\
\hline 6 & 0 & 0 & 0 & $3.0: 10$ & 6.0 & 1700 & 93.34 \\
\hline 7 & 1 & -1 & -1 & $5.5: 10$ & 0.5 & 1100 & 94.20 \\
\hline 8 & -1 & -1 & 1 & $0.5: 10$ & 0.5 & 2300 & 92.98 \\
\hline 9 & 0 & 0 & 0 & $3.0: 10$ & 6.0 & 1700 & 93.20 \\
\hline 10 & 0 & 0 & 0 & $3.0: 10$ & 6.0 & 1700 & 93.05 \\
\hline 11 & 1 & 1 & 1 & $5.5: 10$ & 11.5 & 2300 & 92.98 \\
\hline 12 & -1 & 1 & -1 & $0.5: 10$ & 11.5 & 1100 & 85.88 \\
\hline 13 & 1 & 0 & 0 & $5.5: 10$ & 6.0 & 1700 & 93.71 \\
\hline 14 & 0 & 0 & 1 & $3.0: 10$ & 6.0 & 2300 & 94.26 \\
\hline 15 & 0 & -1 & 0 & $3.0: 10$ & 0.5 & 1700 & 95.05 \\
\hline 16 & 0 & 0 & 0 & $3.0: 10$ & 6.0 & 1700 & 92.84 \\
\hline 17 & 0 & 0 & -1 & $3.0: 10$ & 6.0 & 1100 & 92.42 \\
\hline 18 & 0 & 0 & 0 & $3.0: 10$ & 6.0 & 1700 & 92.88 \\
\hline 19 & 1 & 1 & 0 & $5.5: 10$ & 11.5 & 1700 & 92.21 \\
\hline 20 & -1 & 0 & 0 & $0.5: 10$ & 6.0 & 1700 & 91.78 \\
\hline
\end{tabular}

${ }^{1} X_{1}, X_{2}$ and $X_{3}$ are the ratio of coating material to core material, amount of water added and spray pressure, respectively. 


\section{Results and Discussion}

\section{Optimization of microencapsulation of I. obliquus extract powder by RSM}

To measure the optimal condition of the microencapsulation of I. obliquus extract powder and the relationship between the response (microencapsulation efficiency) and the significant variables, statistical analyses of ANOVA was performed through a joint test of three parameters (Table 3). Among the linear, quadratic and cross-product forms of independent variables, $X_{1}, X_{2}, X_{1} X_{1}$ and $X_{1} X_{2}$ were significant at the level of $p<0.05$. Thus, the regression model equation for the microencapsulation efficiency (\%) could be predicted as follows:

$$
\begin{aligned}
& \text { Microencapsulation efficiency }(\%)=93.4109+1.5610 X_{1} \\
& -1.6880 X_{2}-1.1423 X_{1}^{2}+1.6250 X_{1} X_{2}
\end{aligned}
$$

According to the regression coefficients and $p$-values, the linear term of ratios of core material to coating material $\left(X_{1}\right)$ and amounts of water added $\left(X_{2}\right)$ had significant effects on the microencapsulation efficiency $(p<0.05)$, whereas spray pressure $\left(X_{3}\right)$ had a negligible effect on the microencapsulation efficiency $(p>0.05)$. Therefore, it is indicated in the present study that ratios of core material to coating material and amounts of water added could be the very critical parameters for the microencapsulation of I. obliquus extract powder with high microencapsulation efficiency.

Table 3. Values of regression coefficients calculated for microencapsulation of Inonotus obliquus extract powder using medium-chain triacylglycerol as a coating material

\begin{tabular}{ccccc}
\hline \hline $\begin{array}{c}\text { Independent } \\
\text { variable }\end{array}$ & $\begin{array}{c}\text { Regression } \\
\text { coefficient }\end{array}$ & $\begin{array}{c}\text { Standard } \\
\text { error } \\
\text { coefficient }\end{array}$ & $t$-value & $\begin{array}{c}\text { Significance } \\
\text { level }(p)\end{array}$ \\
\hline Constant & 93.4109 & 0.2532 & 368.985 & 0.000 \\
Linear & & & & \\
$\quad X_{1}$ & 1.5610 & 0.2329 & 6.070 & 0.000 \\
$X_{2}$ & -1.6880 & 0.2329 & -7.249 & 0.000 \\
$X_{3}$ & 0.0600 & 0.2329 & 0.258 & 0.802 \\
Quadratic & & & & \\
$X_{1} X_{1}$ & -1.1423 & 0.4441 & -2.572 & 0.028 \\
$X_{2} X_{2}$ & -0.2573 & 0.4441 & -0.579 & 0.575 \\
$X_{3} X_{3}$ & -0.5473 & 0.4441 & -1.232 & 0.246 \\
Interaction & & & & \\
$X_{1} X_{2}$ & 1.6250 & 0.2604 & 6.241 & 0.000 \\
$X_{1} X_{3}$ & -0.3250 & 0.2604 & -1.248 & 0.240 \\
$X_{2} X_{3}$ & 0.3700 & 0.2604 & 1.421 & 0.186 \\
$r^{2}$ & & & & 0.944 \\
\hline
\end{tabular}

${ }^{1} X_{1}, X_{2}$ and $X_{3}$ are the ratio of coating material to core material, amount of water added and spray pressure, respectively.
The response surface graph for microencapsulation efficiency as a function of two selected parameters $\left(X_{1}\right.$ and $X_{2}$ ) using significant effective factors for the microencapsulation efficiency is shown in Fig. 1 since the spray pressure had not significant effect on the microencapsulation. Increasing the ratio of core material to coating material from $0.5: 10$ to $3.0: 10$ increased the microencapsulation efficiency but the microencapsulation ratio reduced thereafter. An elevation of the amount of water added caused a reduction of microencapsulation.

Response optimization helps to identify the factor settings that optimize a single response or a set of responses. It is useful in determining the operating conditions that will result in a desired response. In the present study, the goal was to search the optimization condition for the encapsulation of I. obliquus extract powder. As shown in Fig. 2, the best combination of factor settings for achiev-

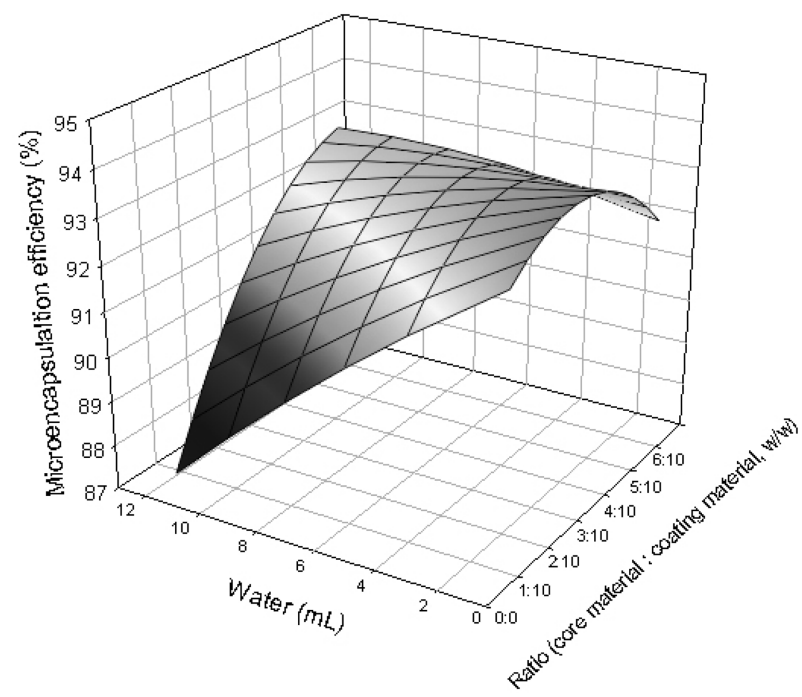

Fig. 1. Response surface for microencapsulation efficiency of Inonotus obliquus extract powder with respect to the ratio of core material to coating material and amount of water added at the spray pressure of $2,300 \mathrm{psi}$.

\begin{tabular}{ccc}
\hline Range & Ratio (coating to core) & Added water $(\mathrm{mL})$ \\
\hline High & $5.50: 10$ & 11.5 \\
Current & {$[2.92: 10]$} & {$[0.5]$} \\
Low & $0.50: 10$ & 0.5 \\
\hline $\begin{array}{c}\text { Microencapsulation } \\
\text { efficiency (\%) }\end{array}$ & \\
y=94.8416 & \\
$\mathrm{d}=0.7667$ &
\end{tabular}

Fig. 2. Process optimization curve for microencapsulation of Inonotus obliquus extract powder. Current means optimum condition of the microencapsulation. $y$ : the yield of the microcapsule, $d$ : desirable score. 
ing the desired response was found to be the ratio of core material to coating material $(2.92: 10)$ and amount of water added $(0.5 \mathrm{~mL})$ with a desirability score of 0.971 which means reaching maximum yield of the capsules.

\section{Microscopic observation}

A photograph of microencapsulated I. obliquus extract produced under the ratio of core material to coating material (3.0:10), amount of water added $(0.5 \mathrm{~mL})$ and spray pressure (1,700 psi) was illustrated in Fig. 3. The overall sizes of microcapsules produced ranged from 1 to $9 \mu \mathrm{m}$. The microscopic examination of microcapsules containing I. obliquus extract revealed spherical particles, and the microcapsules had rounded surfaces and evenly distributed pockets of I. obliquus extract solution in microcapsules made by MCT. According to Choi et al. (2006), the average sizes $(50-200 \mu \mathrm{m})$ of the microcapsules were too sufficiently small to offer a gritty texture to the milk and float to the surface of the milk. In general, homogenized milk has about 2-5 $\mu \mathrm{m}$ in milk fat size, which make milk stable by fat dispersing in milk serum. Based on this phenomena, the capsule size (1-9 $\mu \mathrm{m})$ also evenly disperse in milk, such a small microcapsule size is desirable. Therefore, the textural changes in the food system may be avoided. Finally, the present study showed the sufficiently small size of microcapsules for adding into milk.

\section{Changes in pH, color, and TBA values}

The microcapsules produced under the ratio of core material to coating material $(3.0: 10)$, amount of water added $(0.5 \mathrm{~mL})$ and spray pressure $(1,700 \mathrm{psi})$ for microencapsulation of $I$. obliquus extract powder were added into milk. The changes of $\mathrm{pH}$ in encapsulated or uncap-

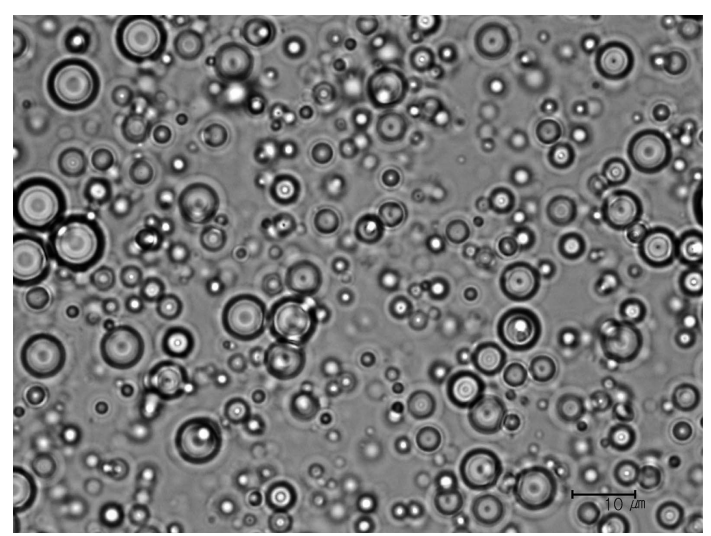

Fig. 3. Photomicrograph of microencapsulated Inonotus obliquus extract with medium-chain triacylglycerol as a coating material. The photograph was taken at $1,000 \times$ magnification. sulated I. obliquus extract-added milk stored at $7^{\circ} \mathrm{C}$ for 6 $\mathrm{d}$ are illustrated in Fig. 4. The $\mathrm{pH}$ values for all the milk samples were nearly constant during 6-d storage and ranged from 6.7 to 6.9 , as a reflective of fresh state (Lee et al., 2006).

Table 4 presents color values of milk samples with the addition of encapsulated or uncapsulated I. obliquus extract during the storage at $7^{\circ} \mathrm{C}$ for $6 \mathrm{~d}$. The L-values significantly reduced with the addition of uncapsulated $I$. obliquus extract during all the storage periods. However, the incorporation of encapsulated I. obliquus extract into the milk samples did not significantly affect the L-values during all the storage periods. Elevating the storage periods from 0 to $6 \mathrm{~d}$ did not significantly influence the Lvalues for all the milk samples studied. According to Philips et al. (1995), the L-value of milk has been demon-

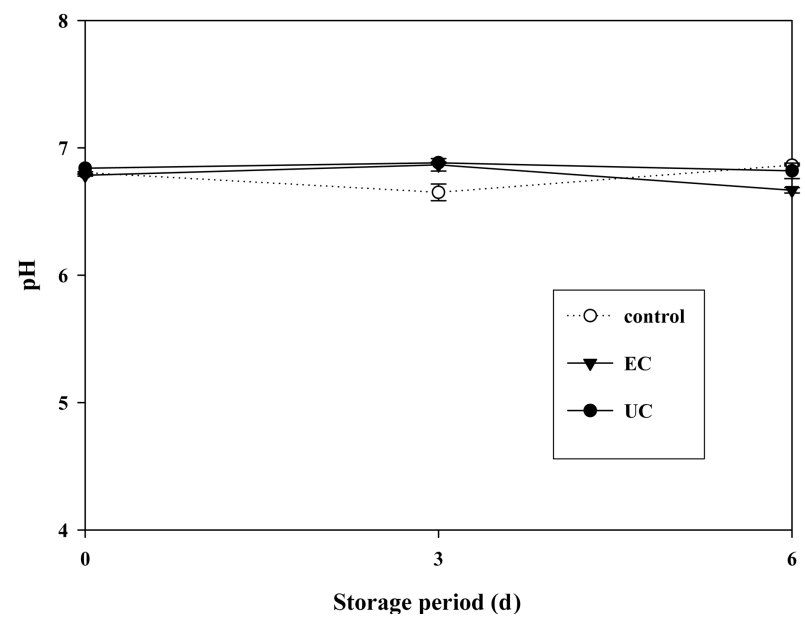

Fig. 4. Changes of $\mathrm{pH}$ in encapsulated (EC) or uncapsulated (UC) Inonotus obliquus extract-added milk stored at $7^{\circ} \mathrm{C}$ during $6 \mathrm{~d}$. Control: market milk.

Table 4. Changes of color for encapsulated (EC) or uncapsulated (UC) Inonotus obliquus extract-added milk stored at $7^{\circ} \mathrm{C}$ during $6 \mathrm{~d}^{1}$

\begin{tabular}{lccrr}
\hline \hline \multirow{2}{*}{ Treatment } & \multirow{2}{*}{$\begin{array}{c}\text { Storage period } \\
(\mathrm{d})\end{array}$} & \multicolumn{3}{c}{ Color value } \\
\cline { 3 - 5 } & & $91.17^{\mathrm{a}}$ & \multicolumn{1}{c}{$\mathrm{a}^{*}$} & \multicolumn{1}{c}{$\mathrm{b}^{*}$} \\
\hline Control $^{2}$ & 0 & $90.65^{\mathrm{e}}$ & $5.61^{\mathrm{d}}$ \\
EC & $-3.06^{\mathrm{d}}$ & $6.08^{\mathrm{c}}$ \\
$\mathrm{UC}$ & & $75.17^{\mathrm{c}}$ & $0.96^{\mathrm{a}}$ & $18.12^{\mathrm{a}}$ \\
\hline Control & & $90.33^{\mathrm{b}}$ & $-3.53^{\mathrm{f}}$ & $6.20^{\mathrm{c}}$ \\
EC & 3 & $90.27^{\mathrm{b}}$ & $-3.04^{\mathrm{d}}$ & $6.32^{\mathrm{c}}$ \\
UC & & $76.71^{\mathrm{c}}$ & $0.62^{\mathrm{b}}$ & $17.38^{\mathrm{b}}$ \\
\hline Control & & $90.66^{\mathrm{ab}}$ & $-3.34^{\mathrm{e}}$ & $5.80^{\mathrm{d}}$ \\
EC & 6 & $90.10^{\mathrm{b}}$ & $-2.84^{\mathrm{c}}$ & $6.22^{\mathrm{c}}$ \\
UC & & $76.42^{\mathrm{c}}$ & $0.72^{\mathrm{b}}$ & $17.35^{\mathrm{b}}$ \\
\hline
\end{tabular}

${ }^{1}$ Values with different letters within the same column differ significantly $(p<0.05)$.

${ }^{2}$ Control: market milk. 
strated to have the most positive impact on consumer appeal. Accordingly, in the present study, it is speculated that the incorporation of the encapsulated I. obliquus extract into the milk samples as well as the prolonged storage (6 d) of the milk samples could not remarkably influence the consumer appeal.

The CIE $a^{*}$ - and $b^{*}$-values slightly increased when encapsulated I. obliquus extract was added into the milk samples. On the other hand, there were remarkable differences between the control and uncapsulated I. obliquus extract-added milk samples during 6-d storage because $I$. obliquus extract powder is originally dark brown. On the basis of all of the results obtained from the present study (CIE L*-, $a^{*}$-, and $b^{*}$ - values), it is suggested that the changes in color values were markedly masked by micro-

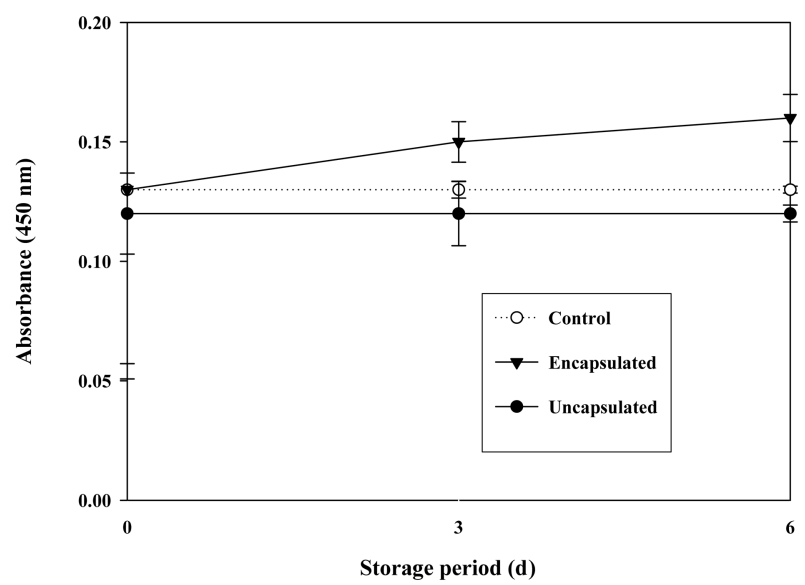

Fig. 5. Changes of thiobarbituric acid (TBA) values in encapsulated (EC) or uncapsulated (UC) Inonotus obliquus extract-added milk stored at $7^{\circ} \mathrm{C}$ during $6 \mathrm{~d}$. Control: market milk. encapsulation of I. obliquus extract powder, even though slight differences were found in color values between the control and encapsulated I. obliquus extract-added milk groups.

The effects of encapsulated or uncapsulated I. obliquus extract addition into milk on chemical oxidation (as measured by the TBA test) during $6 \mathrm{~d}$ of storage at $7^{\circ} \mathrm{C}$ are shown in Fig. 5. TBA absorbance values for the uncapsulated I. obliquus extract-added milk sample was slightly lower than control milk. This result could be explained by that $I$. obliquus extract contains polyphenol which acts as an antioxidant (Ju et al., 2010). In the case of encapsulated I. obliquus extract-added milk samples, the very slight increase in the values of TBA absorbance was found from 0.13 to 0.16 . This result could be explained by that medium-chain triacylglyceride (MCT) used as a coating material may be oxidized during storage of the milk. Therefore, the results indicated that there was no considerable chemical oxidation in the encapsulated or uncapsulated I. obliquus extract-added milk samples during the storage.

\section{Sensory analysis}

The sensory properties of encapsulated or uncapsulated I. obliquus extract-added milk stored at $7^{\circ} \mathrm{C}$ for $6 \mathrm{~d}$ are listed in Table 5. When uncapsulated I. obliquus extract was added into milk at 0 -d storage, brownish scores significantly increased due to the original dark brown color of I. obliquus extract powder. On the other hand, there were no differences in the brownish scores between encapsulated I. obliquus extract-added milk and control samples, confirming the color masking effect of microen-

Table 5. Sensory characteristics of encapsulated (EC) or uncapsulated (UC) Inonotus obliquus extract-added milk stored at $7^{\circ} \mathrm{C}$ during $6 \mathrm{~d}^{\mathbf{1}}$

\begin{tabular}{|c|c|c|c|c|c|c|c|}
\hline \multirow{2}{*}{ Treatment } & \multirow{2}{*}{$\begin{array}{l}\text { Storage period } \\
\text { (d) }\end{array}$} & \multicolumn{6}{|c|}{ Sensory description $^{3}$} \\
\hline & & Brownish & Rancid & Herb flavor & Bitterness & Oily & Overall acceptability \\
\hline Control $^{2}$ & & $1.0^{\mathrm{b}}$ & $1.0^{\mathrm{c}}$ & $1.0^{\mathrm{c}}$ & $1.0^{\mathrm{c}}$ & $1.0^{\mathrm{a}}$ & $5.2^{\mathrm{ab}}$ \\
\hline EC & 0 & $1.5^{\mathrm{b}}$ & $1.3^{\mathrm{bc}}$ & $2.0^{\mathrm{c}}$ & $1.2^{\mathrm{c}}$ & $1.6^{\mathrm{a}}$ & $5.5^{\mathrm{ab}}$ \\
\hline $\mathrm{UC}$ & & $6.0^{\mathrm{a}}$ & $1.2^{\mathrm{c}}$ & $4.8^{\mathrm{a}}$ & $3.7^{\mathrm{a}}$ & $1.8^{\mathrm{a}}$ & $3.8^{\mathrm{b}}$ \\
\hline Control & & $1.0^{\mathrm{b}}$ & $1.0^{\mathrm{c}}$ & $1.0^{\mathrm{c}}$ & $1.0^{\mathrm{c}}$ & $1.0^{\mathrm{a}}$ & $6.0^{\mathrm{a}}$ \\
\hline $\mathrm{EC}$ & 3 & $1.2^{\mathrm{b}}$ & $1.3^{\mathrm{bc}}$ & $1.3 \mathrm{c}$ & $1.2^{\mathrm{c}}$ & $1.7^{\mathrm{a}}$ & $5.3^{\mathrm{ab}}$ \\
\hline $\mathrm{UC}$ & & $5.2^{\mathrm{a}}$ & $2.1^{\mathrm{ab}}$ & $3.3^{\mathrm{b}}$ & $3.0^{\mathrm{ab}}$ & $2.0^{\mathrm{a}}$ & $4.0^{\mathrm{ab}}$ \\
\hline Control & & $1.0^{\mathrm{b}}$ & $1.0^{\mathrm{c}}$ & $1.0^{\mathrm{c}}$ & $1.0^{\mathrm{c}}$ & $1.0^{\mathrm{a}}$ & $5.7^{\mathrm{ab}}$ \\
\hline $\mathrm{EC}$ & 6 & $1.8^{\mathrm{b}}$ & $2.6^{\mathrm{a}}$ & $1.8^{\mathrm{c}}$ & $1.8^{\mathrm{bc}}$ & $2.1^{\mathrm{a}}$ & $4.1^{\mathrm{ab}}$ \\
\hline $\mathrm{UC}$ & & $5.2^{\mathrm{a}}$ & $1.7^{\mathrm{bc}}$ & $3.5^{\mathrm{b}}$ & $2.7^{\mathrm{ab}}$ & $2.2^{\mathrm{a}}$ & $4.8^{\mathrm{ab}}$ \\
\hline
\end{tabular}

${ }^{1}$ Values with different letters within the same column differ significantly $(p<0.05)$.

${ }^{2}$ Control: market milk.

${ }^{3}$ The scale of brownish, rancid, herb flavor, bitterness and oily scores: $1=$ very week, $4=$ moderate, $7=$ very strong. The scale of overall acceptability scores: $1=$ dislike very much, $4=$ either like, $7=$ like very much. 
capsulation. Rancidity and oily scores for the milk samples at 0-d storage were not significantly affected by the addition of encapsulated or uncapsulated I. obliquus extract. Herb flavor and bitterness scores were significantly increased at the entire storage periods when uncapsulated I. obliquus extract powder was used for the production of uncapsulated I. obliquus extract-added milk samples, probably due to the original herb flavor and bitter taste of I. obliquus extract powder. On the other hand, herb flavor and bitterness scores for encapsulated I. obliquus extract-added milk were not significantly different from the control milk sample, indicating masking of the original herb flavor and bitter taste of I. obliquus extract by microencapsulation technique. Overall scores for all the samples studied were not significantly different. Based on all the sensory data obtained from the present study, it is suggested that inappropriate properties of $I$. obliquus extract powder in sensory aspects, such as dark brown color, herb flavor, and bitter taste, can be masked effectively by microencapsulation technique. Thus, the present study indicates the possibility of adding I. obliquus extract powder into milk.

Response surface methodology was successfully used to optimize the microencapsulation conditions for $I$. obliquus extract powder. Based on the results obtained from RSM, the ratio of core material to coating material and amount of water added were the critical parameters to be considered for the microencapsulation of I. obliquus extract powder. The data on the $\mathrm{pH}$, TBA, color, and sensory analysis obtained from the present study indicated that the quality properties of encapsulated I. obliquus extract-added milk were not remarkably different from the control. The production of the milk which incorporates encapsulated $I$. obliquus extract can broaden the utilization of I. obliquus extract powder, and the products can be regarded as possible health-promoting nutraceutical foods.

\section{Acknowledgement}

The present study was supported by a grant of the Brain Korea 21 Project in Seoul, Republic of Korea.

\section{References}

1. Choi, H. J., Ahn, J., Kim, N. C., and Kwak, H. S. (2006) The effects of microencapsulated chitooligosaccharide on physical and sensory properties of the milk. Asian-Aust. J. Anim. Sci. 19, 1347-1353.
2. Cui, Y., Kim, D. S., and Park, K. C. (2005) Antioxidant effect of Inonotus obliquus. J. Ethnopharmacol. 96, 79-85.

3. Fonollá, J., Lopez-Huertas, E., Machado, F. J., Molina, D., Álvarez, I., Mármol, E., Navas, M., Palacín, E., García-Valls, M. J., Remón, B., Boza, J. J., and Marti, J. L. (2009) Milk enriched with "healthy fatty acids" improves cardiovascular risk markers and nutritional status in human volunteers. Nutr. 25, 408-414.

4. Gharsallaoui, A., Roudaut, G., Chambin, O., Voilley, A., and Saurel, R. (2007) Applications of spray-drying in microencapsulation of food ingredients: An overview. Food Res. Int. 40, 1107-1121.

5. Hu, H., Zhang, Z., Lei, Z., Yang, Y., and Sugiura, N. (2009) Comparative study of antioxidant activity and antiproliferative effect of hot water and ethanol extracts from the mushroom Inonotus obliquus. J. Biosc. Bioeng. 107, 42-48.

6. Jeon, B. J., Kim, N. C., Han, E. M., and Kwak, H. S. (2005) Application of microencapsulated isoflavone into milk. Arch. Pharm. Res. 28, 859-865.

7. Ju, H. K., Chung, H. W., Hong, S. S., Park, J. H., Lee, J., and Kwon, S. W. (2010) Effect of steam treatment on soluble phenolic content and antioxidant activity of the Chaga mushroom (Inonotus obliquus). Food Chem. 119, 619-625.

8. Kahlos, K., Kangas, L., and Hiltunen, R. (1986) Antitumor activity of triterpenes in Inonotus obliquus. Planta. Med. 6, 554-556.

9. Kim, N. C., Kim, J. B., and Kwak, H. S. (2008) Microencapsulation of Korean mistletoe (Viscum album var. coloratum) extract and its application into milk. Asian-Aust. J. Anim. Sci. 21, 299-306.

10. Kruger, M. C., Booth, C. L., Coad, J., Schollum, L. M., Kuhn-Sherlock, B., and Shearer, M. J. (2006) Effect of calcium fortified milk supplementation with or without vitamin $\mathrm{K}$ on biochemical markers of bone turnover in premenopausal women. Nutr. 22, 1120-1128.

11. Lee, J. B., Ahn, J., Lee, J., and Kwak, H. S. (2004) L-Ascorbic acid microencapsulated with polyacylglycerol monostearate for milk fortification. Biosci. Biotechnol. Biochem. 68, 495500.

12. Lee, I. K., Kim, Y. S., Jang, Y. W., Jung, J. Y., and Yun, B. S. (2007) New antioxidant polyphenols from the medicinal mushroom Inonotus obliquus. Bioorg. Med. Chem. Lett. 17, 6678-6681.

13. Lee, S. J., Hwang, J. H., Kim, S. H., Min, S. G., and Kwak, H. S. (2006) Comparison of the physicochemical properties of freeze-concentrated versus evaporated milk. Food Sci. Biotechnol. 15, 844-850.

14. Park, Y. K., Lee, H. B., Jeon, E. J., Jung, H. S., and Kang, M. H. (2004) Chaga mushroom extract inhibits oxidative DNA damage in human lymphocytes as assessed by comet assay. Biofactors 21, 109-112.

15. Park, Y. M., Won, J. H., Kim, Y. H., Choi, J. W., Park, H. J., and Lee, K. T. (2005) In vivo and in vitro anti-inflammatory and anti-nociceptive effects of the methanol extract of Inonots obliquus. J. Ethnopharmacol. 101, 120-128.

16. Philips, L. G., McGrief, M. L., Barbano, D. M., and Lawless, 
H. A. (1995) The influence of fat on the sensory properties, viscosity, and color of low fat milk. J. Dairy Sci. 78, 12581266.

17. Sauer, F. D., Farnworth, E. R., Bélanger, J. M. R., Krammer, J. K. G., Miller, R. B., and Yamashiro, S. (1997) Additional vitamin $\mathrm{E}$ required in milk replacer diets that contain canola oil. Nutr. Res. 17, 259-269.

18. Shahidi, F. and Han, X. Q. (1993) Encapsulation of food ingredients. Crit. Rev. Food Sci. 33, 501-547.
19. Stapelfeldt, H., Nielsen, B. R., and Skibsted, L. H. (1997) Effect of heat treatment, water activity and storage temperature on the oxidative stability of whole milk powder. Int. Dairy J. 7, 31-339.

20. Wong, J. Y. and Chye, F. Y. (2009) Antioxidant properties of selected tropical wild edible mushrooms. J. Food Compos. Anal. 22, 269-277.

(Received 2010.6.23/Revised 2010.7.27/Accepted 2010.7.27) 